\title{
Del cálculo diferencial al funcional: consideraciones epistemológicas sobre dos desarrollos históricos"
}

\author{
From differential calculus to functional calculus: \\ epistemological considerations on two historic developments
}

Rafael Andrés Alemañ Berenguer ${ }^{\dagger}$

\begin{abstract}
Resumen
En el presente artículo se ensaya una sucinta comparación entre el desarrollo del cálculo infinitesimal y el de la teoría de distribuciones a fin de extraer conclusiones tanto históricas como epistemológicas. En ambos casos se observa el refinamiento progresivo de ideas intuitivas sugeridas por diversos procesos físicos, hasta convertirse en las bases rigurosas de gran parte de la matemática fundamental. El solapamiento parcial de las investigaciones en cada etapa con la siguiente apoya una visión continuista en el aumento del conocimiento matemático y refuerza la posición del realismo estructural convergente en la filosofía de la naturaleza.

Palabras clave: cálculo infinitesimal - infinitésimos - derivadas - integrales - distribuciones funciones generalizadas - intuición - rigor

Abstract

In the present paper a brief comparison between the development of the infinitesimal calculus and the one of the theory of distributions is attempted in order to draw as much historical conclusions as epistemological ones. In both issues the progressive refinement of intuitive ideas suggested by diverse physical processes is observed, until becoming a rigorous ground for the most of higher mathematics. The partial overlapping of research works in each stage with the following one supports a continuist view for the increase of mathematical knowledge and seems to reinforce the structural-convergence realism point in natural philosophy.
\end{abstract}

Keywords: infinitesimal calculus - infinitesimals - derivatives - integrals - distributions generalized functions - intuition - rigour

\footnotetext{
* Recibido: 28 de Febrero de 2011. Aceptado: 14 de Marzo de 2011.

† Departamento de Física, Ingeniería de Sistemas y Teoría de la Señal (despacho A. Campo-Bagatin), Universidad de Alicante. Para contactar al autor, por favor escriba a: raalbe.autor@gmail.com.

Metatheoria 2(2) (2012): 91-121. ISSN 1853-2322.

(c) Editorial de la Universidad Nacional de Tres de Febrero. Publicado en la República Argentina.
} 


\section{Introducción}

La relación entre la matemática fundamental y las ciencias de la naturaleza -singularmente, la física- constituye uno de los principales puntos de controversia entre quienes han pretendido esclarecer en profundidad el funcionamiento del universo. El curso histórico de los acontecimientos acaecidos en esas disciplinas, sin embargo, muestra un paralelismo que no puede dejar de sorprender a cualquier observador imparcial. No solo ocurre que numerosos progresos matemáticos encuentran una aplicación práctica completamente ajena a las intenciones de sus autores, sino que también en los fenómenos naturaleza suelen encontrarse de modo intrigante las fuentes de inspiración para hallazgos matemáticos cuyas repercusiones alcanzan campos de la matemática sumamente abstractos y alejados de toda experiencia. Argumentar que es la mente humana la que crea un formato abstracto donde encapsular el material bruto de la experiencia resulta menos convincente cuando constatamos que un cierto formalismo encuentra perfecto acomodo donde jamás se espero de él que pudiese hacerlo.

Un magnífico ejemplo histórico de semejante situación lo hallamos en el devenir histórico que conduce desde la invención del concepto de derivada, dentro del marco del cálculo infinitesimal, hasta el nacimiento del más amplio concepto de las funciones generalizadas y sus aplicaciones en el mundo de la física teórica. En ambos casos el desarrollo meramente abstracto corrió parejo a las motivaciones de orden práctico que confluyeron en dos de los hallazgos cardinales de la matemática moderna. Si bien la historia del nacimiento del análisis matemático como tal ha sido tratada con notable amplitud, dada su crucial trascendencia para casi cualquier aspecto del pensamiento científico y filosófico, no ha ocurrido lo mismo con el surgimiento de la teoría de distribuciones, entendido a su vez como un progreso más que hace retroceder las fronteras que limitan la no menos importante idea de función.

A lo largo del presente artículo se examinarán algunos de sus aspectos históricos y epistemológicos.

\section{Los inicios del cálculo}

Inextricablemente unida a las discusiones sobre el concepto de función (Luzin 2003), se hallaban las igualmente delicadas controversias acerca del infinito. Las paradojas de Zenón sobre el movimiento probaron sin duda que había algo equivocado en el entendimiento común de las cantidades infinitas, ya en su vertiente de lo infinitamente grande, como también en la división de magnitudes finitas en una serie infinita de porciones infinitamente pequeñas. Sin embargo, la curiosidad intelectual humana parece igualmente inagotable, y el manejo de las series infinitas -sin pleno rigor- sobrevivió agazapado en el método de exhaución, que a la posteridad legaron Eudoxo de Cnido y Arquímedes de Siracusa (Bochner 1991, p. 259).

La idea de infinito, aunque incómoda, resultaba en la práctica un recurso tan útil en los pasos intermedios de multitud de cálculos matemáticos que difí- 
cilmente nadie pudo prescindir enteramente de ella durante los siglos que mediaron entre la antigüedad clásica y la revolución científica de los siglos XVI y XVII. Dando muestras de una gran perspicacia, Galileo advirtió que algunas partes de un conjunto infinito son tan grandes como el conjunto completo, pero no acertó a distinguir los distintos tipos existentes de cardinales infinitos, lo que sin duda hubiese sido una demanda excesiva. No obstante, muchas de sus estimaciones cuantitativas sobre magnitudes físicas sugieren el empleo de técnicas relativas a las cantidades que después se llamarían "infinitesimales” (Sellés 2006). Pero quienes profundizaron en los problemas prácticos asociados con la obtención de áreas y volúmenes en figuras geométricas, el cálculo de tangentes en curvas diversas, o la manipulación de series infinitas fueron personajes de la talla de Cavalieri, Kepler, Torricelli, Stevin, Fermat, Pascal, Descartes, Roberval, Saint-Vicent, Wallis y Barrow (González-Urbaneja 1992).

Situado ya entre los fundadores del Cálculo, en la figura señera de Gottfried Wilhelm Leibniz (1646-1716) confluyen tanto los métodos geométricos tan en boga en el siglo XVI y XVII como la tradición metafísica del atomismo matemático, todavía vacilante en la difusa frontera entre los "indivisibles" y los "diferenciales". Depositario de un legado intelectual que se remontaba a la antigua Grecia, además de la filosofía y las matemáticas Leibniz cultivó la historia, la jurisprudencia, la lingüística, la teología, la lógica, la diplomacia e incluso la ingeniería. De hecho, ideó una calculadora mecánica, cuya construcción le granjeó su admisión en la Royal Society de Londres a los 27 años.

Leibniz procedió a calcular el área de una región comprendida bajo una curva entre dos puntos $A$ y $B$, imaginándola compuesta de una cantidad infinitamente numerosa de rectángulos infinitesimales, cada uno de ellos con una anchura $d x$ y una altura $y$, donde obviamente $y$ puede variar entre los puntos $A$ y $B$. Mediante lo que denominó "teorema de transmutación”, el sabio alemán obtuvo una técnica general para este tipo de cómputos, la cual encerraba a su vez un anticipo de lo que más tarde se conocería como integración por partes. Esta técnica se suponía basada en la suma de una infinidad de tales rectángulos infinitesimales, por lo que parecía lógico simbolizar la operación alargando la "s" inicial de la palabra latina summa. Así se llega a la familiar notación $\int y d x$, convertida con el tiempo en el emblema del análisis matemático (Child 1920).

Leibniz pensaba en su método de integración ${ }^{1}$ de modo similar a como Cavalieri concebía sus sumas de indivisibles. Imaginando, en la añeja tradición griega, una curva cualquiera como una línea poligonal de infinitos lados, no tuvo dificultad en interpretar dy como la diferencia infinitesimal entre dos ordenadas consecutivas, y $d x$ como la diferencia entre dos abscisas consecutivas. En consecuencia, la operación e $\int y d x$ representaba la suma de una infinidad de pequeños rectángulos infinitesimales caracterizados cada uno de ellos por un área $y d x$. Leibniz también se percató de que el cociente $d y / d x$-la derivada de $y$ con respecto a $x$, en lenguaje moderno- proporcionaba el valor de la tangente en cada punto a

\footnotetext{
${ }^{1}$ Entre 1684 y 1688 Leibniz publicó sus trabajos con el nombre de calculus summatorius. La denominación de cálculo integral fue sugerida en 1690 por Jacob Bernoulli.
} 


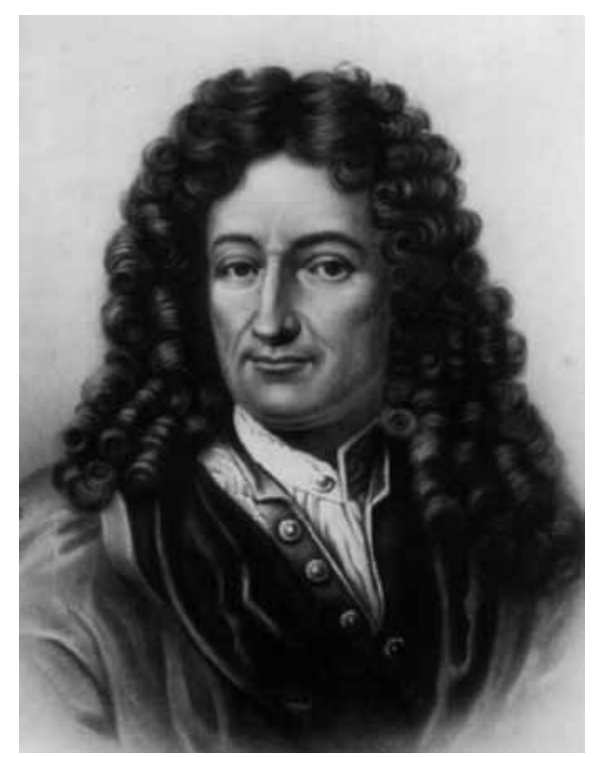

Figura 1. Gorrfried Wilhelm Leibniz

la curva descrita por la relación entre $x$ e $y$, pero no utilizó este resultado como una definición de lo que hoy llamamos "derivada".

A consecuencia de sus trabajos pioneros en el cálculo integral, Leibniz se ve conducido al estudio de unas series infinitas que serán posteriormente conocidas con el nombre de su autor, las "series de Leibniz". Utilizadas como aproximaciones del número $\pi$, estas series convergían a un ritmo desesperadamente lento y además implicaban sustituir la integración de una suma infinita de términos por la suma infinita de integrales, un procedimiento cuyas sutilezas quedarían patentes en los siglos venideros. Más conflictivo quizás fue el hecho de que unos años antes el matemático británico James Gregory (1638-1675) había hallado una aproximación del arco tangente mediante series infinitas de la forma $\operatorname{Arc} \operatorname{tg} x=x-x^{3} / 3+x^{5} / 5-$ $x^{7} / 7+\ldots$, que para $x=1$ se convertía en la serie de Leibniz. Lo cierto es que Gregory nunca dio el paso de sustituir las variables por valores numéricos y no hay duda de que Leibniz desconocía la obra de su colega en las Islas Británicas. ${ }^{2}$

\section{Infinitesimales y fluxiones}

A diferencia de Leibniz, el inglés Isaac Newton (1643 - 1727) desembocó en el inexplorado territorio del Cálculo desde una vía más cercana a la física que a la filosofía. Conocido sobre todo por sus leyes del movimiento y de la gravitación universal, Newton sostenía una opinión peculiar para la época sobre la relación entre la mecánica y la geometría [Newton 1726, prefacio del autor]:

\footnotetext{
${ }^{2}$ En verdad, tampoco Gregory fue el primero en descubrir este resultado. El matemático indio Nilakantha (c. 1444-c. 1544), había expuesto -en verso, por cierto- la existencia de estas series en su obra Tantrasangraha, en 1501 (Roy 1990, pp. 291-306).
} 
Los antiguos consideraron la mecánica en un doble aspecto; como racional, que procede estrictamente por demostración; y práctica. A la mecánica práctica pertenecen todas las artes manuales, de las que la mecánica toma su nombre. Pero como los artesanos no trabajan con perfecta precisión, viene a ocurrir que la mecánica se distingue así de la geometría, que cuanto es perfectamente preciso se llama geométrico; lo que es menos, se denomina mecánico. Pero los errores no residen en el arte, sino en los artífices. Aquel que opera con menos precisión es un mecánico imperfecto; y si alguien pudiese trabajar con precisión prefecta sería el más perfecto de todos los mecánicos [mechanicus omnium perfectissimus]; ya que el trazado de las líneas rectas y los círculos, sobre lo cual se funda la geometría, pertenece a la mecánica. [...] Los trazados de líneas rectas y círculos son problemas, pero no problemas geométricos. La solución de estos problemas conviene a la mecánica; y por la geometría su uso, una vez resueltos, se nos muestra; y es gloria para la geometría que a partir de estos magros principios, de allende aportados, sea capaz de engendrar tanto. En consecuencia, la geometría se funda en la práctica mecánica (Newton 1726, prefacio del autor).

La tradición establecida afirmaba que la mecánica surgía de la cabal aplicación de los postulados geométricos de Euclides al movimiento de los cuerpos materiales. Tales postulados eran auto-evidentes y no necesitaban mayor justificación. Apartándose ostensiblemente de esta concepción, Newton sostenía, muy al contrario, que el conocimiento geométrico nace del estudio de la mecánica como ciencia de los desplazamientos físicos reales. Los axiomas de la geometría confirmaban su validez mediante la observación de los movimientos existentes en la naturaleza, y eran tales movimientos - contra la opinión cartesiana- los que proporcionaban a la geometría su tema de estudio (Burtt 1925, Thayer 1953, Newton 2004, Janiak 2008).

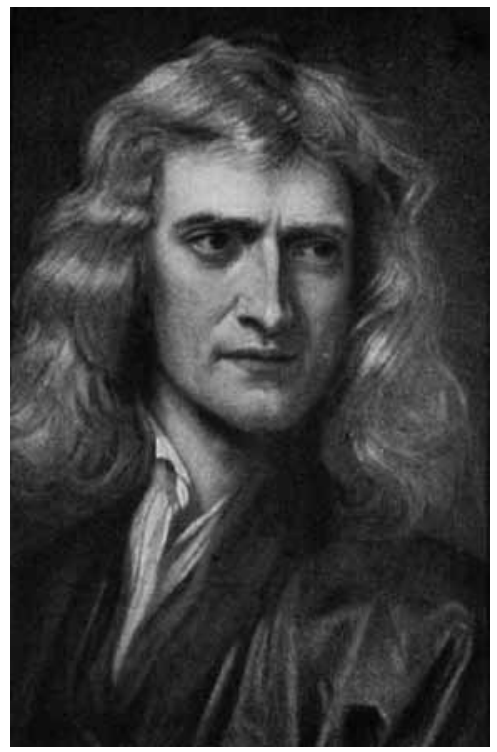

Figura 2. Isaac Newton 
Tras completar en 1666 un tratado sobre su método infinitesimal (Newton 1736), y en 1669 el manuscrito De Analysi sobre series infinitas, publicado en 1711, el genio inglés añadió a estas obras un tratado sobre cálculo y series infinitas, en 1671, y un texto titulado De Quadratura Curvarum en 1693, publicado como anexo en su Opticks once años después. En todas ellas quedaba claro que Newton concebía una curva geométrica como el rastro dejado por un punto móvil, de modo que denominó "fluentes" a las cantidades dependientes del tiempo (posición, velocidad, etc.) -o de cualquier variable independiente distinta del tiempo- y "fluxiones" a sus correspondientes ritmos de variación temporal. Así, para un objeto en movimiento, la posición en función del tiempo, $x(t)$, sería una variable fluente, y la fluxión, $\stackrel{g}{x}(t)$, su variación instantánea que hoy denominamos "derivada". A su vez, las fluxiones eran susceptibles de cambio, permitiendo el cálculo de fluxiones de fluxiones hasta cualquier orden. Newton llamó "momentos" a los cambios infinitesimales de las variables fluentes, en uso de una terminología que cabe comparar con la notación moderna. El momento del tiempo se simbolizaba como o, lo que en la actualidad -según el convenio de Leibniz- se escribiría $d t$. Y el momento del fluente "posición" sería $\stackrel{g}{x}(t)_{0}$; es decir, el producto de la velocidad instantánea por el momento de tiempo, notación que posteriormente evolucionó a $\stackrel{g}{x}(t) d t$.

¿Cómo calculaba Newton una fluxión concreta, y qué significado físico le otorgaba? Supongamos, como hizo Galileo, que el descenso vertical en caída libre de un objeto se muestra proporcional al cuadrado del tiempo transcurrido; esto es, $y \sim t^{2}$, prescindiendo por sencillez de constantes numéricas. La velocidad instantánea de caída se calcularía con el método de fluxiones planteando el cociente de la resta $(t+o)^{2}-t^{2}$, entre el cuadrado del instante $t$ y el cuadrado de un instante posterior $(t+o)$ obtenido incrementado $t$ en una cantidad infinitesimal o. Dicho de otra forma, se trataba de calcular la variación de la fluente durante un tiempo infinitesimalmente breve, y dividirla entre dicho lapso infinitesimal. Empleando para ello el desarrollo del binomio de Newton (el cuadrado de una suma), tenemos:

$$
\dot{y}=\frac{(t+o)^{2}-t^{2}}{o}=\frac{t^{2}+2 t o+o^{2}-t^{2}}{o}=\frac{2 t o+o^{2}}{o}=2 t+o
$$

Tomando igual a cero la cantidad infinitesimal $o$, a todos los efectos, obtenemos ${ }^{3}$ finalmente que $\underset{y}{y} \sim 2 t$. Considerada en sí misma, la fluxión proporciona la ecuación de la recta tangente en un punto a la curva definida por la variable fluente. La operación inversa -obtener la fluente a conocida la fluxión- se llamó "integración”, y en una gráfica suministraba el valor del área comprendida desde el eje horizontal hasta la curva analizada.

Newton era bien consciente del problema entrañado por las magnitudes infinitesimales implicadas en su método de cálculo, que -a semejanza de las dife-

\footnotetext{
${ }^{3}$ Cuando la variable fluente no dependía del tiempo, sino que era una función cualquiera $y=f(x)$, Newton calculaba el cociente $\stackrel{g}{y} / \stackrel{g}{x}$ y al final consideraba la fluxión de $x$ igual a la unidad (el incremento de $x$ en cada unidad de tiempo es uno), lo que se cumple automáticamente para la variable t.
} 
renciales leibnizianas- ni son nulas ni auténticamente finitas. Su respuesta consistía en las denominadas "razones primeras y últimas", toscas antecesoras de los futuros límites matemáticos, como cocientes cantidades "cuasi-nacientes" y "cuasi-evanescentes”. De estas últimas se decía en los Principia con notable oscuridad: "[...] debe entenderse por razón última de cantidades evanescentes la razón de estas cantidades, no antes de desvanecerse, ni después, sino aquella con la cual se desvanecen" (Newton 1997, p. 72).

Como es bien sabido, la carencia manifiesta de bases sólidas en sus métodos analíticos atrajo sobre los primeros calculistas la justificada censura de cuantos filósofos juzgaban estos procedimientos insufriblemente inconsistentes para una disciplina tan sublime como la matemática pura. Entre estos críticos destacó el obispo de Berkeley, quien publicó en 1734 su libro El Analista, o discurso dirigido a un matemático infiel. Donde se estudia si el objeto, los principios y las inferencias del análisis moderno están concebidos con más claridad, o están deducidos de modo más evidente, que los misterios religiosos y los dogmas de la fe. "Primero sácate la viga de tu propio ojo, y entonces verás claramente para sacar la brizna del ojo de tu hermano" (Berkeley 1951).

Como su título indica con todo detalle, Berkeley comparaba la insuficiente fundamentación de los métodos del análisis con la creencia en los misterios de la religión. La diatriba episcopal no se justificaba en el caso de Newton y Leibniz, ambos profundamente creyentes, aunque sí ponía al descubierto las debilidades esenciales de ese nuevo campo de las matemáticas. Newton y Leibniz eran perfectamente conscientes de ello, pero a la vez poseían la lucidez necesaria para advertir que el análisis matemático era una herramienta demasiado poderosa para abandonarla por cuestiones de coherencia fundacional. Y este punto de vista tan pragmático fue también adoptado por los continuadores de su obra, especialmente Leonhard Euler (1707-1783), Jakob Bernoulli (1654-1705) y Johann Bernoulli (1667-1748), quienes ampliaron el repertorio de técnicas para resolver ecuaciones diferenciales de las que habían carecido Newton y Leibniz pero indispensable para los científicos de la Ilustración.

Euler y los Bernoulli concentraron su atención en la búsqueda de soluciones para integrales y series específicas, mostrando menos interés en las propiedades generales necesarias para garantizar la derivabilidad o la continuidad de las funciones. Al igual que sus ilustres predecesores, esta generación de matemáticos carecía aún del rigor necesario para asegurar la validez de muchas de las operaciones que, sin embargo, realizaban con toda soltura. Buen ejemplo de ello era la tendencia a reemplazar la integral de una suma infinita por una suma infinita de integrales; esto es:

$$
\int_{a}^{b}\left[\sum_{k=1}^{\infty} f_{k}(x)\right] d x \rightarrow \sum_{k=1}^{\infty}\left[\int_{a}^{b} f_{k}(x) d x\right]
$$

La integración de funciones y la suma de series implican ambas procesos infinitos que, si se manejan irreflexivamente, pueden conducir con mayor facilidad al 
error que al acierto. Dado que no se ocuparon explícitamente de las condiciones que hacen admisible este intercambio, los pioneros del cálculo -tanto los fundadores como sus sucesores inmediatos- actuaron más guiados por la intuición que por el rigor. Y es un tributo a su genio el hecho de que obtuviesen tantas verdades importantes sobre unas bases tan poco firmes; en especial en el caso de Euler, dotado de una incomparable habilidad para extraer toda la savia de sus argumentos y detenerse justo en la frontera de cuanto la posteridad convalidaría.

Tampoco la generación sucesora de Newton y Leibniz consiguió aclarar la naturaleza de los infinitesimales, que a nadie agradaba su aparente prestancia para ser nulos y no nulos a la vez. El carácter paradójico y antiintuitivo de estos entes no había disminuido por tratarlos como cantidades "evanescentes", un planteamiento dinámico muy propio de alguien -como Newton- primordialmente interesado en el estudio del movimiento. En su Théorie des fonctions analytiques, publicada en 1797, Joseph-Louis Lagrange (1736-1813) trató de fundamentar el cálculo "emancipado de toda consideración sobre cantidades infinitamente pequeñas, cantidades evanescentes, límites y fluxiones” (Lagrange 1813, p. 11). Su estrategia básica consistía en tomar las series infinitas como la fuente primigenia del análisis matemático, y no como objetos abstractos manipulables a posteriori gracias a los procedimientos del cálculo.

En concreto, si comenzamos con una función $f(x)$ de la cual se buscasen sus derivadas, Lagrange desarrollaba la expresión $f(x+i)=f(x)+i p(x)+i^{2} q(x)+i^{3} r(x)+$ ..., donde $p(x), q(x), r(x)$ y los sucesivos coeficientes del desarrollo eran las derivadas del orden correspondiente, obtenidas a partir de la función primitiva $f(x)$ con independencia de la cantidad indeterminada $i$. A diferencia de la series de Taylor, por ejemplo, la formulación de Lagrange situaba en primer lugar las series infinitas y como producto subsiguiente las derivadas. Obviamente, para infortunio del matemático francés no hay garantías de que las series así construidas converjan, ni de que converjan -si lo hacen- a la función de partida. La adecuada cimentación del Cálculo seguía pendiente.

\section{Bolzano, un pionero casi olvidado}

El siguiente paso en el curso histórico que nos atañe viene unido a la obra de un matemático cuyo talento ha recibido menos reconocimiento del que en justicia merecería. Bernard Bolzano (1781-1848) nació en la ciudad bohemia de Praga (actualmente capital de la República Checa) y desde su juventud dio muestras de las aptitudes que posteriormente le permitirían realizar importantes contribuciones a disciplinas tan diversas como la filosofía, la lógica, la matemática y la teología. En 1804 se doctoró en matemáticas con una tesis sobre geometría y después se ordenó como sacerdote católico, lo que facilitó su designación como catedrático de Filosofía y Teología en la Universidad de Praga. Las simpatías mostradas por Bolzano hacia los ideales de la Revolución Francesa motivaron la suspensión de sus funciones tanto por las autoridades civiles como por las religiosas. La censura gubernamental y eclesiástica fue la causa de que la mayoría de las obras de 
Bolzano, quien escribía en alemán, circulasen en forma de manuscritos, cuya escasa difusión no hacía justicia al valor de los trabajos que contenían.

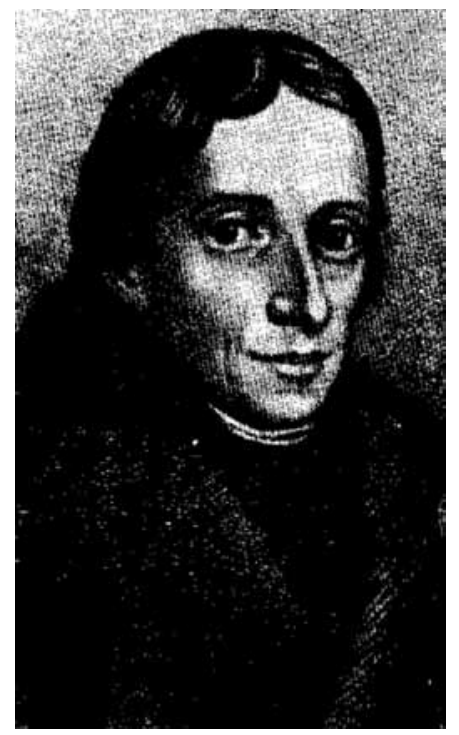

Figura 3. Bernard Placidus Johann Nepomuk Bolzano

La vida de Bolzano coincidió con los inicios del periodo que cabría denominar de "aritmetización" de las matemáticas, un proceso dirigido a reemplazar los elementos empíricos e intuitivos ligados a la geometría que poblaban el pensamiento matemático por un rico repertorio de técnicas simbólicas y procedimientos aritméticos. En concreto, las operaciones aritméticas más generales se vieron libres de la exigencia de una interpretación geométrica asociada. En esta corriente de cambios intelectuales, emergieron numerosas propuestas sobre la caracterización de las nociones de derivada y diferencial. Algunas involucraban el uso explícito de infinitesimales, la utilización de límites sin una correcta definición aritmética o el empleo de series infinitas sin un criterio claro de convergencia.

Pese a todo ello, y a los múltiples intentos de proporcionar las definiciones requeridas, no había entre los especialistas de la época una conciencia de crisis. Los fundamentos del análisis no suscitaban tanta preocupación entre los matemáticos del siglo XVIII como podría suponerse en la actualidad, pues la coherencia lógica se subordinaba a la verosimilitud y al poderío explicativo. Era cierto que no pocas peculiaridades y paradojas aparecían con el uso de infinitesimales y series infinitas, pero nada de eso arrojaba la menor sombra de duda sobre la presunción de veracidad otorgada al núcleo principal del análisis matemático. Esta veracidad venía sugerida tanto por la coherencia global como por el abrumador éxito práctico del Cálculo. La depuración de sus fundamentos, en todo caso, se hacía deseable con objeto de adecuarlo al nuevo ideal de independencia de la matemática de las tradicionales apelaciones intuitivas a cosas tales como cantidades evanescentes, movimientos, o nociones tomadas de la geometría. 
En esta atmósfera intelectual cobran relevancia las opiniones de Bolzano acerca del Análisis, que diferían tanto de sus contemporáneos como de la posteridad. Aunque históricamente apareció al final del camino, desde una perspectiva moderna el desarrollo lógico del análisis matemático ha de comenzar con la definición de número real. Solo después de una correcta definición analítica de los números reales, así como del concepto de continuidad de una función, existe justificación suficiente para deducir los teoremas en que se apoyaban los métodos de resolución de ecuaciones diferenciales en los tiempos de Bolzano. Para él, como para la mayoría de sus contemporáneos, resultaba más importante encontrar métodos de resolución de las ecuaciones que probar las propiedades de continuidad esperables en las funciones tratadas. Tal vez en ello debemos buscar explicación a las dos líneas de pensamiento que separaban las ideas de Bolzano sobre el Análisis de las de sus coetáneos: el categórico rechazo de la idea de infinito en todas sus formas y la estricta separación entre los conceptos aritméticos y geométricos, que a veces llegaba a la supresión de estos últimos.

El infinito podía presentarse a modo de cantidades infinitamente grandes (como el valor de un cociente cuyo denominador se anula o se hace infinitamente pequeño), infinitamente pequeñas (según opinaba Newton, algo inferior a cualquier cantidad concebible aunque distinto de cero), o colecciones infinitamente numerosas (como el conjunto de todos los números naturales). Bolzano considera que la definición de infinitésimo como cantidades «en la práctica menores que cualquier [...] cantidad concebible es contradictoria" (Bolzano 1817, Prefacio). En el caso particular del Cálculo, Bolzano da por sentado que «debe ser sabido por todos que [las reglas] pueden expresarse de tal modo que el concepto de lo infinitamente pequeño (que de otro modo sin duda se asociaría con las expresiones $d x, d y, d z$ ) pueda ser completamente evitado" (Bolzano 1817, Prefacio). Y posiblemente no era esta una opinión minoritaria entre sus colegas, quienes solían rechazar al unísono los límites y los infinitesimales. ${ }^{4}$ Por fortuna, Bolzano no se mostraba tan refractario a estas ideas, ya que decidió conservar como concepto básico el límite (aunque sin usar esa palabra), en tanto rehusaba el uso de los infinitésimos.

Según se ha señalado antes, la otra característica distintiva del pensamiento de Bolzano sobre el Análisis era la finura con que discriminaba entre los argumentos aritméticos y los geométricos. Para un autor como el británico Isaac Barrow, inmerso en pleno siglo XVII, los problemas principales en los inicios del Cálculo eran de naturaleza geométrica, de donde cabía esperar que hallasen su más apropiada solución por medios geométricos. Durante el siglo XVIII en el continente europeo, la formulación algebraica de tales problemas -asistida por la más cómoda notación de Leibniz y por el refinamiento del concepto de fun-

\footnotetext{
${ }^{4}$ Por ejemplo, el texto de Lagrange publicado en 1797 se titulaba Teoría de las funciones analíticas conteniendo los principios del cálculo diferencial, prescindiendo de toda consideración sobre infinitésimos, evanescentes, limites y fluxiones, y reducidos al análisis algebraico de cantidades finitas.
} 
ción- pasó de ser percibida como una mera descripción conveniente de una situación mecánica o geométrica a constituir un cuerpo teórico independiente susceptible de recibir a posteriori una interpretación mecánica o geométrica. Con el fin de asegurar la independencia entre sus vertientes aritmética y geométrica, era esencial que el Análisis no tomase préstamo alguno de la geometría a menos que pudiese reformularlo en términos puramente aritméticos, una condición de la cual Bolzano siempre fue plenamente consciente:

Nada quiero mencionar aquí sobre los defectos en el álgebra superior y el cálculo diferencial e integral. Es bien sabido que hasta ahora no se ha dado acuerdo alguno acerca del concepto de diferencial. Solo a finales del año pasado la Regia Sociedad Jablanovsky de Ciencias en Leipzig ofreció como tema de su galardón la [...] discusión de las diferentes teorías del cálculo infinitesimal y la decisión sobre cuál de ellas resulta preferible. (Bolzano 1810, Prefacio)

Bolzano, como con otros muchos autores, ha sufrido la tendencia de sus comentaristas en años sucesivos (Birkhoff 1973) a interpretar sus logros en términos de los usos y estilos de la matemática muy posterior, por ejemplo con respecto a sus ideas sobre el concepto aritmético de límite o acerca de la convergencia de las series infinitas. Para eludir este peligro se hace imperativo distinguir cuidadosamente entre la definición de un concepto y la formulación simbólica de ese concepto que permite operar con eficacia en el marco de la teoría general donde se emplea. El uso efectivo de un nuevo concepto requiere una visión amplia del contexto general o del marco teórico en cuyo seno ese nuevo concepto poseerá conexiones claras con otras ideas ya bien establecidas, y en consecuencia vinculaciones nítidas con los problemas existentes en esa teoría.

A semejanza de la mayoría de sus colegas, Bolzano asociaba mentalmente el paso al límite con un proceso infinito, o con el recurso a cantidades infinitamente minúsculas, lo que él rehuía con vehemencia (Rusnock 2000, p. 63, y Russ 1980, p. 179). Y lo mismo podría decirse de su trabajo sobre la convergencia de series infinitas. El hecho es que la propia noción de "variable" y la transición desde las cantidades variables en sentido físico a las variables abstractas de la lógica y la matemática pura requirieron un trabajo de aquilatación que se extendió durante décadas a través de no pocas vacilaciones, aciertos y descarríos. En sus trabajos más tempranos, Bolzano había desarrollado ya una gran destreza al emplear cantidades arbitrariamente diminutas de modo equivalente al procedimiento de los límites para demostrar resultados típicos sobre derivadas (Grattan-Guinness 1970a, 1970b y Russ 2006). Uno de ellos fue la constatación de que la diferenciabilidad de una función en un punto implicaba su continuidad en ese mismo punto. Y resultó un testimonio impresionante de su planteamiento conceptualista de las matemáticas que unos cuantos años después hubiese profundizado en los conceptos de función, continuidad y derivada hasta el extremo de probar que, contrariamente a las expectativas sugeridas por la intuición, la afirmación inversa -que la continuidad implicaba diferenciabilidad- era falsa. 


\section{La consolidación de los fundamentos}

Finalmente el francés Augustin-Louis Cauchy (1789-1857) sí obtuvo eco cuando determinó que para una cantidad variable cualquiera expresada como sucesión de números reales, el límite se definía como un valor fijo al que se aproximaban indefinidamente los valores sucesivamente atribuidos a la mencionada variable, de modo que acaben por diferir de él tan poco como se quiera (Cauchy 1821, 1823). Y con este nuevo entendimiento también se iluminó sobre el concepto de derivada, rigurosamente definible mediante los límites matemáticos:

El origen de la teoría de Cauchy de la derivada se asemeja en algunos aspectos a la de los conceptos de límite, continuidad y convergencia. Tal como había hecho con los conceptos de límite y suma de una serie, Cauchy tomó el viejo concepto de cociente diferencial, o derivada, y le dio un nuevo y preciso significado. Definió la derivada al estilo del siglo dieciocho como el límite de la razón del cociente de diferencias, pero la definición estaba basada en su nueva y clara comprensión del concepto de límite. Recuérdese que Cauchy había definido el límite como sigue: "Cuando los valores atribuidos sucesivamente a la misma variable se aproximan indefinidamente a un valor fijo, hasta que acaba por diferir de él tan poco como se desee, este último es llamado el límite de todos los demás”. (Grabiner 1981, p. 114)

Sin embargo, la noción de límite en Cauchy todavía se interpretaba como un proceso secuencial de aproximaciones sucesivas que tienen lugar consecutivamente a lo largo de un cierto tiempo finito. Con ello se asocia el paso al límite de una variable con cantidades en movimiento con un flujo de valores asignado a la magnitud cuyo límite se calcula, es decir, con una cantidad en movimiento al modo newtoniano (Fisher 1978 y Grabiner 1978, 1981). De este error, así como la confusión entre funciones derivables y funciones continuas, se ocupó el alemán Karl Wilhelm Theodor Weierstrass (1815-1897), quien aritmetizó definitivamente el análisis, liberándolo de cualquier reminiscencia referida al cambio temporal o a proceso dinámico alguno en el seno de las operaciones matemáticas.

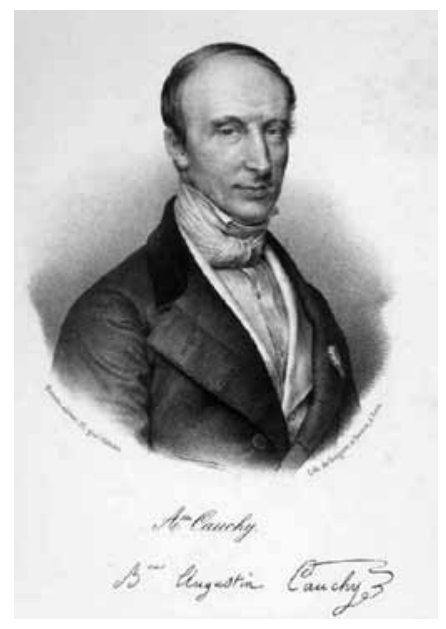

Figura 4. Augustin Louis Cauchy 
Empleando la notación actual, sea una sucesión $a_{n}$ cuyos subíndices pertenezcan al conjunto de los números naturales $N$. La sucesión se dice convergente a $L$, y $L$ se denomina entonces el límite de $a_{n}$, si $\forall \varepsilon>0, \exists m \in N|\forall i>m \rightarrow| a_{i}-\mathrm{L} \mid<\varepsilon$. En el caso más simple referido a las funciones, tengamos una función $f$ definida en un entorno $\Omega=\left\{x ;\left|x-x_{0}\right|<\rho ; \rho>0, \rho \in \mathbb{R}\right\}$ de $x_{0}$. Se dice que $S$ es el límite de $f$ en $x_{0}$ si $\forall \varepsilon>0, \exists \delta>0, \delta<\rho|\forall x| x-,x_{0}|<\delta \rightarrow| S-f(x) \mid<\varepsilon$. Weierstrass proporcionó también criterios rigurosos de convergencia para las series infinitas, ya manejadas por Newton y Leibniz sin mucho rigor, que habían quedado fuera del alcance de Cauchy (Boyer 1959).

Hacia 1873, casi un siglo tras la desaparición de Euler y dos tras el inicio del análisis matemático, las dudas suscitadas por Berkeley parecían definitivamente disipadas. Sin embargo, como si tratasen de compensar los temores exorcizados, en las postrimerías del siglo XIX los matemáticos convocaron nuevos espantos desde el reino de la continuidad y la derivabilidad. El más famoso de ellos acaso sea la función patológica de Weierstrass, continua en todos sus puntos y derivable en ninguno (Weierstrass 1895, pp. 71-74), cuya revelación disgustó profundamente a estudiosos de la talla de Hermite, Poincaré y Picard, aferrados a una intuición geométrica a punto de abandonar el centro del escenario intelectual. Johannes Karl Thomae (1840-1921) presentó en 1875 la función "regla", continua en los puntos irracionales pero discontinua en los racionales (Thomae 1875, p. 14). Y eso no es todo, pues la función $U(x)=\left\{x^{2} \operatorname{sen}(1 / x)\right.$ si $x \neq 0$ y 0 si $\left.x=0\right\}$ proporciona un ejemplo de que la derivada de una función diferenciable no necesariamente ha de ser continua.

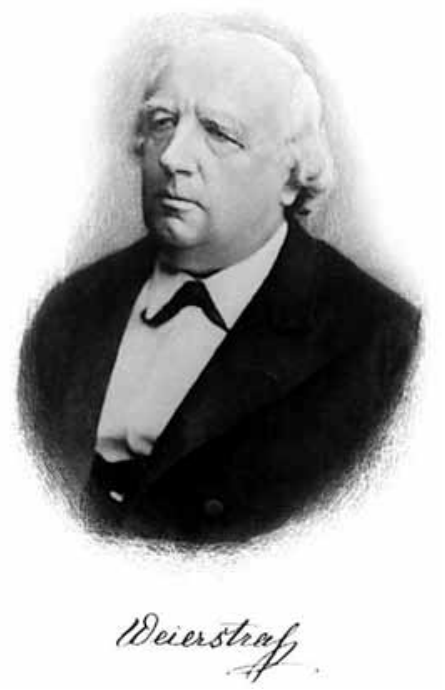

Figura 5. Karl Theodor Wilhelm Weierstrass

Otra cuestión delicada surgía al considerar el caso de una serie de funciones $f_{k}$ que convergen puntualmente a una función $f$. La pregunta, ya examinada por 
Weierstrass, era si el carácter continuo de todos los miembros de la serie $\left\{f_{k}\right\}$ permite asegurar que $f$ también debe ser continua. La respuesta es negativa, de lo que se deduce que una propiedad compartida por todos los miembros de una serie no siempre ha de ser poseída por el límite -si existe- de dicha serie. Incluso cuando la serie $\left\{f_{k}\right\}$ converge puntualmente, digamos, no cabe inferir en general que sea susceptible de intercambiar el límite de la integral por la integral del límite:

$$
\lim _{k \rightarrow \infty}\left[\int_{a}^{b} f_{k}(x) d x\right]=\int_{a}^{b}\left[\lim _{k \rightarrow \infty} f_{k}(x)\right] d x
$$

Weierstrass demostró que para el intercambio de límites e integrales la convergencia uniforme es condición suficiente pero no necesaria. Es decir, pueden hallarse ejemplos de series $\left\{f_{k}\right\}$ que convergen puntualmente pero no uniformemente, y sin embargo satisfacen la igualdad (3). En los umbrales del siglo XX todos estos resultados, a la vez sorprendentes e inquietantes, pusieron ante los ojos de los matemáticos la conveniencia de reconsiderar la propia idea de función.

\section{Algo más que funciones}

Las controvertidas propiedades de las series infinitas y de sus límites revistieron una importancia creciente debido a los trabajos del físico-matemático francés Joseph Fourier (1768-1830), cuyas series homónimas estaban llamadas a desempeñar un papel crucial en el desarrollo del Cálculo. Estudiando la ecuación que rige la propagación del calor, Fourier halló un método para la descomposición de funciones periódicas en series trigonométricas convergentes, método posteriormente ampliado mediante procedimientos integrales (las integrales de Fourier) al caso de funciones no periódicas (Bottazzini 1986, p. 72). En el curso de las manipulaciones formales aplicadas sobre estas series e integrales, Fourier hizo un uso explícito de variables que asumían valores infinitamente grandes e infinitamente pequeños, confiando -acaso con demasiado optimismo- en que los procesos de paso al límite las justificarían (Fourier 1822, p. 417). El alemán Johann Peter Gustav Lejeune Dirichlet (1805-1859) contribuiría algo más tarde a esclarecer la situación (Dirichlet 1830) determinando las condiciones bajo las cuales las series e integrales de Fourier adquirían pleno sentido.

Pero el trabajo de Dirichlet había revelado que algunas de las cantidades utilizadas por Fourier no podían juzgarse funciones genuinas, aun cuando jugasen un papel esencial en la validez de resultados. Insatisfechos con los manejos de Fourier, Cauchy y su compatriota Siméon Denis Poisson (1781-1840) se basaron en la modelización matemática de ciertos fenómenos físicos para introducir factores multiplicativos auxiliares $h(s)$ y $h_{k}$ en las integraciones y los sumatorios respectivamente. De ese modo atribuían con notable liberalidad -especialmente Cauchy- valores finitos a integrales divergentes (Laugwitz 1989). Incluso Weierstrass, bien reputado por su rigor matemático, dejó entreveradas sus obras con funciones tan peculiares como $\delta(k)=(4 \pi k)^{-1 / 2} \exp \left(-x^{2} / 4 k\right)$, por la cual una función continua $f(x)$ cumpliría (Weierstrass 1988, p. 25): 


$$
\int \delta(x-t) f(t) d t \approx f(x)
$$

La expresión (4) posee para las funciones continuas el equivalente de la propiedad "selectora" típica de, por ejemplo, la delta de Kronecker, $\delta_{i j}$. En efecto, cuando de una serie de elementos $\left\{a_{j} \mid j \in \mathrm{N}\right\}$ deseamos entresacar un $a_{i}$ en concreto, basta con aplicar $\sum_{j} \delta_{i j} a_{j}=a_{i}$. Como es bien sabido, $\delta_{i j}=1$ si $i=j$, y $\delta_{i j}=0$ si $i \neq j$, con la condición de normalización $\sum_{i j} \delta_{i j}=1$. Fourier mismo era consciente de esta propiedad selectora presente en la serie $1 /(2 \pi)+(1 / \pi) \Sigma \cos n\left(x-x_{0}\right)$, con $n$ $\in\left[1, \infty\left[\right.\right.$, en el intervalo fundamental $\left(x-x_{0}\right) \in[-\pi, \pi]$.

Este tipo de aplicaciones poco rigurosas eran ya de uso corriente entre físicos e ingenieros tanto por su comodidad práctica como por su marcado carácter intuitivo. Tomemos una unidad de masa distribuida unidimensionalmente sobre un intervalo $[-L, L]$ a lo largo de un segmento de longitud total $\ell$, según la función de densidad $\rho(x)$. Dentro de dicho intervalo $\int \rho(x) d x=1$, y fuera de él $\rho(x)=0$. Imaginando ahora que el valor de $L$ va reduciéndose paulatinamente, la distribución lineal de masa colapsará en $x=0$ cuando $L$ se anule. En el límite de este proceso, la densidad se hará infinita en $x=0$ puesto que toda la masa se concentraría en un punto sin longitud, y no obstante se diría intuitivamente que la cantidad total de masa sigue siendo la misma - una unidad- pese a encontrarse concentrada de modo tan peculiar. Aunque no muy ortodoxo, se trata de un procedimiento usual en la interpretación de propiedades físicas definidas sobre medios continuos (Demidov 2001, pp. 1-2).

Tratamientos similares resultaban muy comunes en situaciones gobernadas por operadores laplacianos, ya en gravitación o en electrodinámica, como la ecuación genérica de Poisson, $\nabla^{2} \phi \propto-\rho$, donde $\phi$ y $\rho$ son respectivamente el potencial y la densidad de masa o de carga eléctrica. ${ }^{5}$ No es de extrañar, así pues, que fuesen autores relacionados con las ciencias naturales, como el alemán Gustav Robert Kirchhoff (1824-1887) y el británico Oliver Heaviside (1850-1925), quienes intentasen ofrecer una definición más nítida de las pseudofunciones $\delta$. Kirchhoff se vio conducido a este terreno por su interés en las soluciones de la ecuación tridimensional de ondas (Kirchhoff 1878, 1882), mientras que Heaviside abordó la cuestión a través del cálculo operacional (Heaviside 1893, 1894 y Lützen 1979). Sin mucho rigor para el punto de vista moderno, Heaviside especuló con la posibilidad de identificar $\delta(x)$ con la derivada de la función "escalón”, o función de Heaviside, ${ }^{6} H(x)$, una de cuyas versiones se define como aquella cuyo valor es 1 para $x>0,1 / 2$ si $x=0$, y 0 para $x<0$.

En la década de 1920 la física cuántica constituía la primera línea del frente en la investigación de la estructura íntima de la materia. Y en esa bulliciosa atmósfera

\footnotetext{
${ }^{5}$ En las ciencias aplicadas, y particularmente en ingeniería, las influencias sobre un sistema muy localizadas en el espacio o el tiempo -los "pulsos"- se representan mediante una $\delta$. A su vez el modelo formal de la respuesta del sistema suele darse en términos de la función denominada "propagador de Green", $G\left(x, x^{\prime}, t\right)$, representativa de la transmisión de una señal desde $x$ a $x^{\prime}$ en un tiempo $t$.

${ }^{6}$ Una introducción muy accesible para lectores de campos científicos distintos de la matemática profesional se contiene, por ejemplo, en Levine (1977, pp. 173-174).
} 
correspondió al físico británico Paul Adrien Maurice Dirac (1902-1984) conceder un decisivo protagonismo a estos extraños operadores ${ }^{7}$-por él manipulados como contrapartidas continuas de la delta de Kronecker- que desde entonces pasarían a conocerse como "deltas de Dirac". Con los límites de integración en los extremos del intervalo abierto $]-\infty,+\infty\left[\right.$, se cumplía la igualdad $\int \delta(x) d x=1$, además de la condición $\delta(x)=0$ para $x \neq 0$, lo que dejaba bien clara la índole atípica de esta magnitud:

\begin{abstract}
$\delta(x)$ no es una función de $x$ de acuerdo con la definición matemática usual de función, la cual requiere que una función tenga un valor definido para cada punto en su dominio, sino que es algo más general, que podemos llamar 'función impropia' para manifestar su diferencia con respecto a una función definida del modo usual. Así, $\delta(x)$ no es una cantidad que en general pueda utilizarse en el análisis matemático como una función ordinaria, por cuanto su empleo ha de confinarse a ciertos tipos simples de expresiones para los cuales es obvio que no puede surgir inconsistencia alguna. [...] aunque una función impropia no tiene en sí misma un valor bien definido, cuando aparece como un factor en el integrando la integral posee un valor bien definido. [...] Por tanto debería ser posible reescribir la teoría en una forma en la cual las funciones impropias aparezcan por entero en los integrandos únicamente. El uso de funciones impropias, pues, no involucra carencia de rigor alguna en la teoría, sino que meramente es una notación conveniente que nos faculta para expresar de manera concisa ciertas relaciones que podríamos transcribir, en caso necesario, a una formulación que no entrañase funciones impropias, aunque tan solo de un modo enrevesado que tiende a oscurecer el argumento. (Dirac 1958, pp. 58-59)
\end{abstract}

La razón de todo ello estribaba en la necesidad de generalizar las nociones al uso en los espacios vectoriales lineales cuando la dimensión de tales espacios se hacía infinita. ${ }^{8}$ En cualquier espacio vectorial lineal de dimensión $n$ los componentes de una base ortonormal $\left\{e_{i} \mid i, j=1,2, \ldots, n\right\}$ cumplen la relación $e_{i} \times e_{j}=\delta_{i j}$. Cuando se efectúa la transición a espacios de dimensión infinita puede ocurrir que la base contenga una cantidad infinita no numerable de elementos, de modo que ya no cabe escribir $e_{i}$ con $i$ un índice discreto, sino más bien $e(\xi)$ con $\xi$ una variable continua. La condición de ortonormalidad vendría dada ahora por e $(\xi) \times e\left(\xi^{\prime}\right)$ $=\delta\left(\xi-\xi^{\prime}\right)$; por eso sustituyó Dirac la delta de Kronecker por la suya propia. La teoría cuántica representa el estado de un sistema físico mediante lo que se denomina "vector de estado", el cual pertenece en general a un espacio funcional, también de índole vectorial lineal, llamado espacio de Hilbert. En este contexto las variables típicas de la dinámica clásica ceden su puesto a operadores que actúan sobre los elementos de estos espacios de Hilbert, donde los autoestados de algunos subconjuntos de estos operadores forman las bases ortonormales permitidas en dichos espacios de Hilbert. Los postulados cuánticos establecen que

\footnotetext{
${ }^{7}$ Curiosamente, si consideramos que Poisson fue uno de los primeros en utilizar una versión pionera de las futuras deltas de Dirac, también es cierto que Dirac reformuló los corchetes de Poisson de la mecánica clásica como conmutadores de los operadores correspondientes en la teoría cuántica (Dirac 1926, p. 663).

${ }^{8}$ Los espacios funcionales suministran un ejemplo muy común de dimensionalidad infinita. Pensemos, por ejemplo, en los polinomios de Legendre $\left\{P_{\ell}(x)\right\}$ donde $x \in[-1,1]$ y $\ell=0,1,2, \ldots, \infty$.
} 
cualquier medida de una cierta propiedad física revelará que el sistema se halla en uno de tales autoestados. La importancia de las deltas de Dirac se hace patente cuando advertimos que con frecuencia muchos de estos operadores cuánticos (posición, momento lineal, energía) poseen conjuntos continuos de autovalores (Schwartz 1968).

\section{El significado de la delta de Dirac}

Dada una función $f$ continua y de buen comportamiento (no "patológica" en el sentido de Weierstrass), con valores bien definidos en cada punto de su domi-

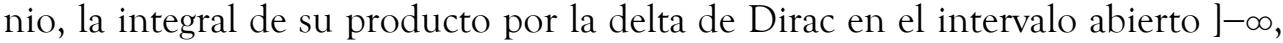
$+\infty$ [, dependía tan solo del valor que tomase cuando su argumento fuese 0 . En palabras del científico inglés:

La propiedad más importante de $\delta(x)$ está ejemplificada por la siguiente ecuación

$$
\int_{-\infty}^{+\infty} f(x) \delta(x) d x=f(0)
$$

donde $f(x)$ es cualquier función continua de $x$. [...] El lado izquierdo [de esta ecuación] solo puede depender de valores de $f(x)$ muy cercanos al origen, de manera que podemos reemplazar $f(x)$ por su valor en el origen, $f(0)$, sin un error esencial. (Dirac 1958, p. 59)

De esta circunstancia deduce Dirac en esa misma página una igualdad equivalente a la obtenida por Weierstrass el siglo anterior, es decir:

$$
\int_{-\infty}^{+\infty} f(x) \delta(x-a) d x=f(a)
$$

No carece de importancia insistir en las diferencias que separaban los estilos metodológicos de quienes se entregaron a fundamentar la naciente física cuántica. Weyl empleó la teoría de grupos para esclarecer las bases de la teoría, en tanto Wigner utilizaba esas mismas técnicas concentrándose en sus aplicaciones físicas. Von Neumann, por su parte, desarrolló un formalismo basado en los espacios de Hilbert con el propósito de que el carácter probabilista de la teoría emergiese en él de forma natural. Sin embargo, Dirac siempre manifestó el deseo de conceder primacía al razonamiento físico: "[...] la matemática es solo una herramienta y deberíamos aprender a conservar las ideas físicas en nuestras propias mentes sin referencia a la forma matemática" (Dirac 1958, p. viii).

Esta preponderancia de la racionalidad física sobre la matemática -digámoslo así- acaso sea disculpable en un físico, aunque sugiere también unos interrogantes nada desdeñables. En primer lugar, puesto que la $\delta(x)$ no es estrictamente una función, Dirac no podía estar seguro de que al incluirla en una integral obtuviese un resultado con sentido, ya fuese físico o matemático. Las investigaciones llevadas a cabo durante más de un siglo nos brindan buenas razones para su- 
poner que las funciones ordinarias se comportan adecuadamente en el contexto del cálculo integral, pero no había razones para aventurar eso mismo en el caso de las funciones impropias. A Dirac tan solo le quedaba abrigar una expectativa de que así fuese, y comprobarlo con el uso, como de hecho ocurrió.

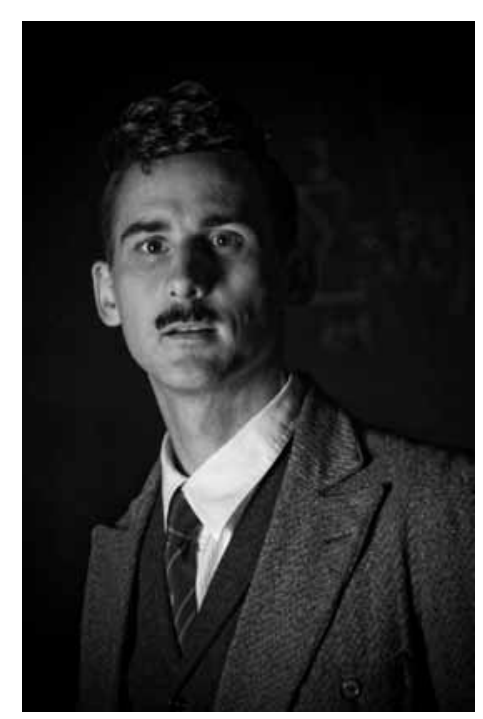

Figura 6. Paul Adrien Maurice Dirac

Otra curiosa circunstancia tiene que ver con el hecho de que la $\delta(x)$ carece de valor definido en cualquier punto de su dominio, y pese a ello se define su integral sobre dicho dominio. Es más, el valor no nulo de esta integral parece surgir de un modo muy poco claro, pues resulta extremadamente peculiar que el valor de una integral sea no nulo en un único punto. La respuesta de Dirac a esta objeción fue que la delta "se anula en todas partes excepto dentro de un pequeño dominio, de longitud $\varepsilon$ digamos, alrededor del origen $x=0$. [No obstante, el valor de la función] es tan grande dentro de este dominio que su integral sobre este entorno es la unidad" (Dirac 1958, p. 58). Por ello, en sentido estricto, el valor no nulo de la integral no proviene de un único punto, sino de un pequeño entorno en la inmediata vecindad de ese punto.

Con todo, cabe preguntarse si de veras existe alguna función -propia o impropia- que satisfaga este requisito, y de ahí que Dirac subraye que el formalismo de la $\delta(x)$ es susceptible de reformularse prescindiendo de funciones impropias aunque al precio de una mayor complejidad. Así lo hizo von Neumann (1932, pp. 3-33) cuando señaló que, con el fin de establecer la equivalencia entre la mecánica ondulatoria y la matricial, la delta de Dirac servía para garantizar que todos los operadores autoadjuntos pudiesen adoptar una forma diagonal. Previamente a esa discusión, este mismo autor aseveraba:

No hay objeción aquí [a la introducción de conceptos matemáticos inconsistentes, como la delta de Dirac] si estos conceptos, que no pueden ser inclui- 
dos en el marco actual del análisis, fuesen intrínsecamente necesarios para la teoría física. Por tanto, tal como la mecánica Newtoniana dio lugar primero al desarrollo del cálculo infinitesimal, que, en su forma original, era indudablemente no auto-consistente, así la mecánica cuántica podría sugerir una nueva estructura para nuestro "análisis de una cantidad infinita de variables" -p.e., la técnica matemática habría de cambiarse, y no la teoría física. (von Neumann 1932, p. ix).

Nótese que en la cita precedente, von Neumann reconoce el paralelismo existente entre el caso histórico del cálculo infinitesimal en la mecánica clásica y su posterior fundamentación, con el uso en la teoría cuántica de las deltas de Dirac, cuya consistencia matemática habría de esperar aún casi dos décadas para probarse. Y el curso a seguir parece ser el mismo (salvo un notable acortamiento de los tiempos hasta la consolidación formal), no solo porque los argumentos físicos van a la cabeza del avance explorando el significado físico del formalismo matemático, sino también porque también en este caso el estudio de un determinado ámbito de la naturaleza inspira el desarrollo de toda una nueva área de la matemática fundamental, cuyo desenvolvimiento y ramificaciones nadie pudo pronosticar.

\section{Distribuciones y funciones generalizadas}

A mediados del siglo XX las matemáticas estaban preparadas para una teoría satisfactoria de funciones generalizadas. Su necesidad se veía acrecentada por los sucesivos intentos de definir derivadas de funciones que no eran derivables, como las funciones escalonadas, generalmente para resolver ecuaciones diferenciales. Por ejemplo, el cálculo simbólico de Heaviside para resolver la ecuación diferencial ordinaria de los circuitos eléctricos, y las partes finitas de Hadamard, introducidas para obtener fórmulas explícitas de lo que hoy llamamos soluciones fundamentales de la ecuación de ondas en varias variables.

A consecuencia de ello, la utilización masiva de las deltas de Dirac por los físicos, y en multitud de cálculos por los ingenieros, acicatearon todavía más a quienes en la comunidad matemática urgían la búsqueda de una justificación rigurosa para tales manejos. Se reproducía con ello, en cierta medida, la situación sobrevenida tras la invención del cálculo infinitesimal por Newton y Leibniz, cuando los problemas de fundamentación quedaron relegados en espera de mejores tiempos ante el éxito práctico de un método en apariencia desprovisto de rigor. El primer paso firme en esa dirección se debió a Salomon Bochner (1899. 1982) a comienzos de la década de 1930 (Bochner 1932), seguido por Sergei Lvovich Sobolev (1908-1989), quien caracterizó en 1935 las $\delta(x)$ como una clase especial de funcionales (Lützen 1982). De hecho, fue Sobolev, en la segunda mitad de la década de 1930, el matemático que más se aproximó a la definición de una distribución. Este notable matemático ruso definió las distribuciones de orden finito como funcionales lineales y continuos, pero nunca consideró el espacio $D$ de las funciones infinitamente diferenciables con soporte compacto con la topología adecuada. Las aportaciones de Sobolev fueron muy importantes en la 
resolución del problema de Cauchy para ecuaciones lineales hiperbólicas con un número impar de variables espaciales. Sin embargo, nunca mencionó la delta de Dirac $\delta(x)$, la convolución o la conexión con la transformada de Fourier.

La definitiva formalización llegaría entre 1950 y 1951 de la mano del francés Laurent Schwartz (1915-2002), con su Teoría de Distribuciones, que se convertiría en un clásico de la matemática en el siglo XX (Schwartz 1950-1951). Las herramientas matemáticas necesarias para una teoría unificada de funciones generalizadas estaban esencialmente disponibles en la década de 1940, gracias a la publicación de la monografía Théorie des Opérations Linéaires de Banach en 1932. Además los miembros del grupo Bourbaki, con los que Schwartz estaba en contacto, eran expertos en la teoría de la dualidad en espacios localmente convexos. Según el propio Schwartz, la definición de distribución como un funcional lineal y continuo sobre el espacio $D$ de las funciones infinitamente diferenciables con soporte compacto, dotado de la topología del límite inductivo, le fue sugerida por su trabajo anterior sobre la dualidad en los espacios de Fréchet, y por su conocimiento -gracias al grupo Bourbaki- de la teoría de medidas de Radon como funcionales sobre el espacio de las funciones continuas con soporte compacto. La gestación definitiva de sus ideas no se produjo sin dificultades, pero finalmente el gran matemático francés dio con las ideas que andaba buscando:

\begin{abstract}
Schwartz desarrolló estos conceptos y varios teoremas sobre sus operadores en una noche de octubre de 1944. En los seis meses siguientes continuó trabajando sobre el mismo tema. Alrededor de febrero de 1945, comenzó a desarrollar una teoría de trasformadas de Fourier para sus operadores, pero se encontró con grandes dificultades. Intentó resolverlas durante varios meses. Por fin un día, de repente, se percató de que todos sus problemas se resolvían fácilmente si definía las funciones generalizadas no como operadores, sino como funcionales secuencialmente continuos [...]. A estos nuevos objetos los llamó distribuciones [...]. Según sus propias afirmaciones, la definición "correcta" de distribución le fue sugerida a Schwartz por los dos hechos siguientes: 1) Su trabajo anterior sobre la dualidad de espacios de Fréchet, 2) Su conocimiento, a través del grupo Bourbaki, de la teoría de medidas de Radon, en particular la $\delta$, que podían representarse como funcionales sobre un espacio de las funciones continuas con soporte compacto. (Bombal 2003, p. 193)
\end{abstract}

El soporte de una función $x: \mathrm{R} \rightarrow \mathrm{C}$ del conjunto de los números reales en el conjunto de los complejos, se define como el cierre topológico $\overline{\mathrm{A}}$ del conjunto $\mathrm{A}$ $=\{t \in \mathrm{R} \mid x(t) \neq 0\}$. Dado que el soporte ya se define como cerrado, bastará que sea acotado para ser compacto. Una función con soporte compacto es, entonces, aquella cuyo soporte se encuentra contenido en un intervalo cerrado. Una función de prueba es entonces una función infinitamente diferenciable con soporte compacto. El conjunto de las funciones de prueba se denota por $T$.

Así pues, una distribución es una transformación lineal $F: T \rightarrow C$, y se dice que es una distribución continua si $F\left(x_{k}\right) \rightarrow 0$ en C siempre que $x_{k} \rightarrow 0$ en T. La familia de todas las distribuciones viene denotada por $D$ y es claro que constituye un espacio vectorial sobre C. Dada una función integrable $f: \mathrm{R} \rightarrow \mathrm{R}$, podemos definir la distribución $F$ dada por 


$$
F(x)=\int_{-\infty}^{\infty} f(t) x(t) d t=\int_{a}^{b} f(t) x(t) d t(7)
$$

donde $[a, b]$ contiene al soporte de $x \in T$. La linealidad de la integral indica que se tiene una distribución, y además continua, por aplicación directa de la definición. Se dice entonces que la función genera la distribución. Una distribución generada por una función integrable se denomina regular, y en caso contrario recibe el nombre de distribución singular. Tenemos entonces que toda distribución regular es continua.

Como se dijo en apartados anteriores, en sentido estricto una función con las propiedades atribuidas a la delta de Dirac no existe. Pero, de existir, podríamos definir la distribución "regular" generada por tal $\delta$ como:

$$
F(x)=\int_{-\infty}^{\infty} \delta(t) x(t) d t=x(0) F(x)=\int_{-\infty}^{\infty} \delta(t) x(t) d t=x(0)
$$

Por la discusión anterior, no existe tal distribución regular, pero aún podemos definir la distribución singular dada por $F(x)=x(0)$, a la que llamaremos con toda propiedad la "distribución Delta de Dirac".

Lo que de hecho había conseguido Schwartz era crear un cálculo que extendía la clase de las funciones ordinarias a una nueva clase de objetos abstractos, las distribuciones, los cuales todavía conservaban algunas de las operaciones básicas del análisis, como la diferenciación o la adición y la multiplicación por funciones pertenecientes al conjunto $\mathrm{C}^{\infty}$. Tal como fue definido por Schwartz, el espacio de las distribuciones consiste en funcionales lineales continuos sobre $\mathrm{C}^{\square}$, es decir, el espacio dual del espacio de las así llamadas funciones de prueba, provisto de una topología dada que comporte la convergencia de las derivadas de todos los órdenes (Halperin \& Schwartz 1952, Brezis \& Browder 1998).

Bajo la denominación de "funciones generalizadas" y en la línea de Scwhartz," estos objetos matemáticos se definen como funcionales lineales continuos, a los que se extienden apropiadamente las operaciones de diferenciación y transformación de Fourier. No deja de ser interesante plantearse la cuestión de hasta qué punto cabría identificar con legitimidad las deltas de Dirac con las distribuciones de Scwhartz, ya que las primeras fueron reconocidas por su autor como una técnica operatoria fundamentalmente inconsistente, mientras las distribuciones gozan de la misma consideración que cualquier otra rama de la matemática bien consolidada. Tal vez para acallar estas dudas se abrió la posibilidad de adoptar un planteamiento más cercano al de Dirac, contemplando la $\delta(x)$ como el límite de una cierta sucesión infinita de funciones diferenciables ordinarias (Gel'fand \& Shilov 1964). Este procedimiento guarda una estrecha semejanza con la definición de los números irracionales como el límite de una sucesión de Cauchy de números racionales. Las funciones generalizadas pueden considerarse asimismo como condiciones de contorno impuestas sobre funciones analíticas en el eje real, empleando el

\footnotetext{
${ }^{9}$ Aunque en este trabajo no se hará, cabe distinguir entre las distribuciones, como elementos de un espacio de Schwartz, y las funciones generalizadas como elementos de un espacio de Colombeau (Egorov 1990, p. 3). Otras extensiones interesantes de la teoría pasan por el análisis no estándar de Robinson (Lightstone \& Wong 1975, pp. 759-762).
} 
poderoso arsenal de las transformaciones de Fourier en el plano complejo bien conocidas en la solución de ecuaciones diferenciales parciales (Bremermann \& Durand 1961 y Carmichael \& Mitrovic 1989). Incluso es posible desarrollar una visión ampliada de las distribuciones de Schwartz mediante avanzadas técnicas de álgebra y topología, dando justificación al cálculo operacional de Heaviside (Mikusińnki 1983).

Recibida al principio con reticencias por un considerable número de matemáticos que no acababan de confiar en tan exóticos objetos, la teoría de distribuciones fue ganando adeptos paulatinamente. Buena parte de las posiciones ganadas se obtuvieron gracias al fervor del mismo Schwartz, quien no cejaba en su encendida defensa (Gårding 1991, p. 79): "El éxito inicial de la teoría se debió a una mercadotecnia activa y entusiasta, a la convicción de Laurent Schwartz sobre la importancia de las distribuciones, y a las ardorosas conferencias que pronunció en diversos países europeos tras la guerra”. Sin duda, haber recibido en 1950 la Medalla Fields en el Congreso Internacional de Matemáticas de Harvard ayudó en la tarea. La difusión de la teoría en la entonces Unión Soviética quedó a cargo del eminente académico Israel Moiseevich Gel'fand (1913-2009) y de sus colaboradores, con resultados más que notables.

En Rusia, las distribuciones fueron presentadas en una serie de seis atractivos volúmenes acerca de "Funciones Generalizadas" publicados por Gel'fand, Shilov y sus coautores a partir de 1958. Estos libros no tuvieron un gran eco en Occidente, pero enfatizaron la influencia de los espacios localmente convexos en análisis matemático. A pesar del éxito cosechado por las distribuciones de Schwartz, al principio la teoría fue acogida con escepticismo y notable reticencia entre algunos matemáticos (Gårding 1991).

La teoría de distribuciones fue un gran paso hacia las aplicaciones del análisis funcional abstracto. Muchos de los resultados acerca de distribuciones y de sus aplicaciones al análisis de Fourier o las ecuaciones en derivadas parciales no se podrían haber demostrado, o siquiera conjeturado, sin el auxilio de sofisticadas técnicas de análisis funcional. Aunque de muchos de ellos se encontraron luego pruebas puramente analíticas, el análisis funcional despeñó un papel capital en su descubrimiento. La teoría de distribuciones y sus aplicaciones y el análisis funcional abstracto se han influido mutuamente de modo muy beneficioso en campos tan diversos como la representación de grupos de Lie, las corrientes de De Rham en variedades, la teoría cuántica de campos, o el análisis armónico y las ecuaciones en derivadas parciales.

\section{Antecedentes y repercusiones del trabajo de Schwartz}

Con la aparición en 1932 del libro de John von Neumann Mathematische Grundlagen der Quantenmechanik y de Linear Transformations in Hilbert Spaces and Applications in Analysis, de Marshall Stone, la teoría de operadores (en espacios 
de Hilbert) se había consolidado como un área de investigación por derecho propio, aunque íntimamente relacionado con lo que se conoce ahora por análisis funcional lineal, que en aquellos años experimentó su primer gran desarrollo. ${ }^{10}$

Fue también en los años treinta y principios de los cuarenta cuando las fronteras del análisis funcional comenzaron a extenderse hasta que las fronteras de esta disciplina llegaron a difuminarse. Las investigaciones de Gel'fand en la estructura de álgebras de Banach conmutativas reunificaron la teoría general del análisis funcional lineal con la teoría de operadores para dar lugar, entre otras cosas, al análisis de Fourier en grupos abelianos localmente compactos y con ello al nacimiento del análisis armónico.

Después de la Segunda Guerra Mundial, la escuela francesa de análisis funcional prosiguió la labor que la escuela polaca había iniciado y desarrolló una serie de investigaciones intensivas sobre la estructuras de los espacios vectoriales topológicos (especialmente sobre los espacios de funciones diferenciables y sus duales). Supongamos que se tiene un espacio vectorial $V$ real o complejo, en cuyo seno cabe definir una topología. Cuando esta topología verifica ciertas propiedades en relación con las operaciones de $V$ se dice que tenemos un espacio vectorial topológico. Los espacios normados son un caso particular de los e.v.t. (y a su vez los espacios de Hilbert un caso particular de los normados).

Fue en este marco de esfuerzos matemáticos cuando Laurent Schwartz introdujo la teoría de distribuciones, tras la cual se produjo el descubrimiento, por Bernard Malgrange y Leon Ehrenpreis, de un resultado capital: toda ecuación en derivadas parciales homogénea con coeficientes constantes tiene solución distribucional fundamental (Tréves 1966). Así se explica que la búsqueda de fundamentos rigurosos para un objeto como la delta de Dirac no fue un caso particular, sino parte de un programa de una mayor perspectiva dentro del cuadro de la matemática de esa época.

En la teoría de distribuciones de Schwartz, se toma un abierto $\Omega$ del espacio euclídeo $n$-dimensional y se considera el espacio vectorial sobre el cuerpo de los números complejos de las funciones complejas definidas en $\Omega$, infinitamente diferenciables y de soporte compacto. A este espacio se le llama $D(\Omega)$ y se le da una estructura lineal topológica. Los elementos del dual topológico de $D(\Omega)$ son las distribuciones de Schwartz. No obstante, salvo ciertas distribuciones, como pueden ser las funciones localmente integrables o algunas medidas de Radon, como la delta de Dirac, en general, la existencia de distribuciones se apoya en el teorema de extensión de Hahn-Banach que, a su vez, utiliza el axioma de elección de Zermelo.

La teoría de las distribuciones de Schwartz transformó, a partir de entonces, muchas áreas del análisis, y supuso un gran avance en la teoría de ecuaciones en derivadas parciales. Como se ha visto, Schwartz asimiló muchas ideas y descubrimientos de las décadas precedentes (de Heaviside, Sobolev, Bochner y otros)

\footnotetext{
${ }^{10}$ Con el establecimiento, por ejemplo, de tres enunciados fundamentales: el teorema de extensión de HahnBanach, el teorema de Banach-Steinhaus y el teorema de la aplicación abierta.
} 
y añadió otras nuevas, en especial la transformada de Fourier de distribuciones atemperadas y el teorema de los núcleos (Schwartz 1954-1955, 1957, 1959).

Las distribuciones difundieron los múltiples usos de la dualidad y el modo de superar los límites de los espacios de Banach, inadecuados para tratar ciertas cuestiones. Introducidas en principio para manipular soluciones singulares de una ecuación, las distribuciones consiguen abordar satisfactoriamente por separado las cuestiones de regularidad por una parte y de existencia y aproximación por otra. En ese sentido, la mayor contribución de Schwartz a la formalización de su teoría -además del teorema de los núcleos- fue la decisión de tomar el espacio de las funciones de decrecimiento rápido y su dual de las distribuciones atemperadas como el marco adecuado para el análisis de Fourier (Tréves 1966). La teoría de las distribuciones exhibe así como uno de sus logros principales la facultad de utilizar la transformada de Fourier en condiciones mucho más amplias que las permitidas hasta entonces. Con la teoría de funciones generalizadas se repitió en cierto modo el proceso de generalización de la noción de función que hizo posible el desarrollo del análisis de Fourier. Las distribuciones, por otra parte, también pusieron de manifiesto el papel central de la convolución en el análisis armónico y en sus conexiones con las ecuaciones en derivadas parciales.

\section{Consecuencias epistemológicas}

A semejanza del período que discurre desde Newton y Leibniz hasta Cauchy y Weierstrass, la evolución histórica entre Dirac y Schwartz invita a interesantes reflexiones epistemológicas. Una primera característica del trabajo de los descubridores del Cálculo, compartida con los creadores de las funciones generalizadas, es la motivación inicialmente pragmática de sus esfuerzos. La inspiración provenía del deseo de matematizar un cierto proceso de la naturaleza, ya fuese mediante la determinación de parámetros mecánicos instantáneos (velocidad, aceleración) o expresando la relación entre los operadores diferenciales e integrales en la física cuántica. En ambos casos, los instrumentos formales disponibles en cada época se revelaron insuficientes y fue una mezcla de audacia y genialidad la que condujo a sus respectivos autores a la elaboración del cálculo infinitesimal y de las funciones impropias.

Tras la primera etapa, en la que predominaron claramente motivaciones de índole física, vino un segundo estadio dirigido hacia una más rigurosa fundamentación de tales procedimientos. Varias generaciones más tarde las investigaciones de diversos expertos desembocaron en el concepto aritmético de límite y la aclaración definitiva de la idea de continuidad en matemáticas. Por su parte, tan solo unos años después de su aparición en el marco de la teoría cuántica, las distribuciones o funciones generalizadas vinieron a rescatar las deltas de Dirac de la inconsistencia lógica.

En la fundamentación de ambas ramas de la matemática jugó un papel esencial la idea de serie ordenada, e incluso precisando aún más, la noción de "orden". Un conjunto ordenado se dice bien ordenado cuando todo subconjunto 
no vacío de dicho conjunto tiene un primer elemento. Se dice que dos conjuntos bien ordenados tienen el mismo número ordinal si existe una semejanza entre ellos, es decir, una aplicación biyectiva que conserve el orden. La aritmética ordinaria considera números cardinales finitos, lo cual explica que a cada número cardinal viene a corresponder un solo número ordinal. Por esta razón, nunca se construyó una aritmética de números ordinales pues, de haberse intentado, se habría obtenido una mera copia de la aritmética ordinaria. Sin embargo, para ordinales infinitos el caso es muy diferente, como se constata por el hecho de que el conjunto de los números naturales admite una infinidad de ordenaciones para las cuales resulta bien ordenado, de tal manera que los números ordinales correspondientes son distintos (por eso, la aritmética transfinita de Cantor fue algo nuevo en su tiempo, que produjo un gran impacto). A finales del siglo XIX no se sabía si cualquier conjunto se podría ordenar bien, ni siquiera se sabía si el conjunto de los números reales tendría esta propiedad. Esto condujo a la definición de los números aleph. Un aleph es el número cardinal de un conjunto que se pueda ordenar bien. Más tarde, utilizando el axioma de elección, Zermelo demostró que todo conjunto puede ser bien ordenado o, lo que es lo mismo, que todo número cardinal es un aleph (Zermelo 1904).

Ya se expuso en el apartado correspondiente cómo Weierstrass purgó definitivamente el concepto de límite de cualquier vínculo con un proceso infinito de cambio de una variable con el tiempo. En su momento constituyó uno de los más notables logros del pensamiento matemático, y como tal debe ser tenido todavía hoy. Por eso mismo, llama poderosamente la atención el hecho de que la pulimentación de las bases lógicas en que se fundaban las funciones impropias de Dirac, que permitió la transición a las funciones generalizadas, encontró una de sus más diáfanas vías también en la idea de serie ordenada, precisamente a través de un paso al límite de una serie formada por funciones.

En efecto, en términos de la función "escalón" de Heaviside, $\mu(x-a)$, cuyo valor se toma 0 si $x<a$ y 1 si $x>a$, definimos la función $h_{\varepsilon}(x-a)=[\mu(x-a+\varepsilon / 2)-\mu(x-a$ $-\varepsilon / 2] 1 / \varepsilon$. Entonces, para todo $\varepsilon>0$,

$$
\int_{-\infty}^{+\infty} h_{s}(x-a) d x=\int_{a-\varepsilon / 2}^{a+s / 2} \frac{1}{\varepsilon} d x=1
$$

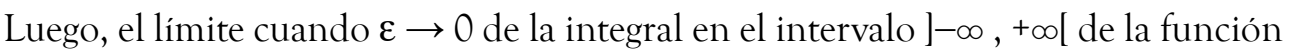
$h_{\varepsilon}(x-a)$ es igual a 1 .

Así parecería que el concepto de orden es al menos tan importante en la matemática fundamental como el de cantidad, aunque este último -gracias a la idea de número- siempre haya sido considerado el ingrediente imprescindible de todo razonamiento matemático. Los defensores del logicismo, a comienzos del siglo XX, llegaron a plantearse incluso si era posible reformular la mayoría de las operaciones matemáticas sin necesidad de recurrir al concepto de número. Finalmente no fue posible por las razones que uno de sus más reputados autores expuso: 
Parece natural preguntar: ¿No podríamos pasarnos sin coordenadas de ningún género, ya que estas han llegado a ser poco menos que denominaciones convencionales sistemáticamente aplicadas? Quizás esto sea posible con el tiempo; pero, al presente, carecemos de los conocimientos necesarios para ello. Deseamos, por ejemplo, poder diferenciar, pero no podemos diferenciar una función a menos que sus argumentos y valores sean números. [...] Asimismo podemos definir una función "continua" no numérica [...]. Lo que en cambio no ha sido definido, excepto con el auxilio de los números, es una fracción. Ahora bien, $d y / d x$ es el límite de una fracción; así, aunque podamos generalizar la noción de límite, no nos es posible actualmente generalizar la expresión $d y / d x$ porque no podemos generalizar la definición de fracción. $\mathrm{Pa}$ rece claro a priori que, puesto que la diferenciación de coordenadas es físicamente útil, incluso cuando el valor cuantitativo de dichas coordenadas es convencional, debe existir algún proceso, del cual la diferenciación es una forma numérica especial, que pueda aplicarse siempre que tengamos funciones continuas, hasta en el caso de que no sean numéricas. La definición de un proceso tal es un problema en lógica matemática, probablemente soluble, pero hasta ahora no resuelto. (Russell 1969, pp. 87-88)

Tal vez las propiedades de ordinalidad y cardinalidad resulten mutuamente irreducibles, como parece indicar la intrigante indiscernibilidad cuántica. El hecho de que en un sistema cuántico sea posible definir un número cardinal para el conjunto de sus elementos pero no sea -al menos en parte- una ordenación de los mismos por carecer estos de identidad individual quizás origine una nueva fuente de inspiración para futuros avances matemáticos.

Otra de las reflexiones que suscita este transcurso histórico entra de lleno en el papel de las matemáticas en la construcción de las teorías físicas, pues se argumenta con asiduidad que resulta imposible expresar los principios físicos fundamentales de la naturaleza -especialmente en la teoría cuántica- sin recurrir a un depurado lenguaje matemático. Y ya que las entidades matemáticas se revelan indispensables para nuestras mejores teorías sobre la realidad físicas, prosigue el argumento, nos vemos conducidos a creer en su existencia (Colyvan 2001).

El impresionante aluvión de situaciones en las que una técnica matemática inicialmente desligada de todo uso práctico encontró una aplicación idónea en cierta rama de la física solo tiene parangón en los casos -igualmente numerososen los que cierto fenómeno natural sugirió la idea de una estructura abstracta cuya formalización posterior desveló regiones insospechadas de la matemática. Y entre todos esos casos destacaban los aquí analizados en relación con el cálculo infinitesimal y las funciones generalizadas. No significa esto que nos vemos obligados a adoptar un compromiso ontológico equivalente para los entes físicos y los matemáticos. Tal confusión supondría olvidar la paciente labor de los autores que durante años se han entregado a desenmarañar los posibles significados del concepto de existencia. La existencia conceptual de las entidades matemáticas, lejos de postular una modalidad existencial autónoma, permite manipularlos por medio de prescripciones, definiciones y estipulaciones. Se trata de entes que, pese a su inmaterialidad, existen objetivamente -o mejor, intersubjetivamentecon las mismas características básicas para las mentes de todos los seres materiales que los manejan. Los constructos u objetos conceptuales no son entidades 
materiales, ni forman parte del mundo físico; por el contrario, son elementos de nuestra representación abstracta del mundo (Bunge 2006). Los constructos no existen sin procesos cerebrales que los elaboren.

Pero esta distinción tan pertinente no puede oscurecer un interrogante sustancial, como es la relación entre las estructuras formales de la matemática abstracta y las regularidades empíricamente observables en el mundo material. A la luz de la evidencia acumulada, no durante años sino durante siglos, parece difícil sustraerse al veredicto de que nuestras representaciones abstractas del mundo material no son puramente arbitrarias sino que de algún modo reflejan o participan de unas pautas que existen objetivamente en la naturaleza. De ahí los abrumadores éxitos logrados por el programa de matematización de la filosofía natural (debería decirse "mecanización" en sus primeros tiempos), emprendido desde los tiempos de Galileo y Newton. Si ello es así -y, con todo, deberíamos mostrar una extrema cautela en esto- surgiría un dilema de difícil solución: ¿es la realidad esencialmente formalizable matemáticamente o tan solo captamos aquellos aspectos de la naturaleza susceptibles de matematización de modo que el resto permanecerá por siempre más allá de los límites de lo humanamente cognoscible? Incluso la propia formulación de estos interrogantes causa un vértigo metafísico cuya respuesta, o el esclarecimiento mismo de si existe tal respuesta, no se vislumbra cercana.

\section{Conclusiones}

Un recorrido comparativo entre los desarrollos respectivos del cálculo infinitesimal y de las funciones generalizadas, aun tan breve como el precedente, sitúa en su justa perspectiva un proceso histórico que no deja de suscitar interesantes reflexiones. En primer lugar, comprobamos que el repetido tópico según el cual los científicos de la naturaleza acuden en busca de instrumentos formales al repertorio dispuesto por los matemáticos puros opera no pocas veces al contrario. Con apreciable asiduidad son los fenómenos naturales los que inspiran operaciones y conceptos abstractos que acaban encontrando un asiento privilegiado en el concierto de los objetos matemáticos. Suele recurrirse con frecuencia al ejemplo de la teoría de grupos, desde sus primeros balbuceos en los papeles póstumos del malogrado Évariste Galois (1811-1832) hasta su refinada aplicación en las más elegantes teorías de partículas elementales. También contamos con casos de fertilización cruzada; el cálculo tensorial comenzó su andadura gracias a los estudios de las tensiones en medios materiales de Woldemar Voigt (1850-1919) y después se convirtió en una poderosísima rama de la matemática gracias a Gregorio RicciCurbastro (1853-1925) y Tullio Levi-Civita (1873-1941). Poco después, los tensores recuperaban su preeminencia en la física guiados por Albert Einstein (1879. 1955), quien los utilizó como armazón de su teoría general de la relatividad.

Los métodos infinitesimales, con todas las salvedades que se quiera, siguieron en buena medida un camino inverso. En manos de Newton las derivadas se concibieron como "fluxiones", flujos o ritmos de cambio de variables describien- 
do trayectorias que por la pura fuerza del intelecto se figuraban casi tan tangibles como las estelas de los guijarros arrastrados por una corriente de agua. Los "indivisibles" de Cavalieri, trasunto matemático de las mónadas leibnizianas, se convirtieron en cantidades evanescentes bajo la pluma del genio de Woolsthorpe. Un siglo después de las vacilaciones de Newton y Leibniz, los trabajos de Cauchy y Weierstrass -sin olvidar al brillante Bolzano- dieron carta fundacional a operaciones que ya eran irrenunciables en las matemáticas y en las ciencias naturales.

Además de la inextricable conexión entre la matemática pura y el mundo natural, tan misteriosa hoy como siempre, aparece de nuevo un rasgo del quehacer científico no por repetido menos olvidado. La historia de las ciencias que tan aseadamente recogen numerosos manuales, plena de investigadores resueltos a proceder con pleno rigor sin alejarse un ápice de los senderos de la lógica más estricta, se muestra pura quimera en confrontación directa con los hechos históricos. Las investigaciones - mejor aun, los investigadores- avanzan a tientas por terrenos desconocidos sin otra brújula que la intuición ilustrada y el talento que a cada uno quepa desplegar.

No siempre su rigor carece de fisuras, ni su intuición resulta infalible; pero cuando apresan una pieza de valor no renuncian a ella meramente por desconocer sus entresijos más recónditos. Newton y Leibniz fundaron el cálculo infinitesimal y merecen la eterna admiración de la humanidad aunque no supiesen fundamentarlo sin reservas. Esa fue tarea de las generaciones posteriores, las cuales, a su vez, prosiguieron socavando las certezas de sus predecesores al ampliar la idea de función hasta desembocar en el concepto de "función generalizada".

Otra de las conclusiones que puede colegirse de la comparación entre ambos decursos históricos nos recuerda la tan a menudo olvidada importancia del concepto de orden, así como la relativa dispensabilidad de los conceptos de "número" o "cantidad". Como se ha mencionado, el entrejuego de la aritmética ordinal y la cardinal, disimulado en los conjuntos finitos, cobra toda su importancia en el dominio de los transfinitos cantorianos. Y, dado que la noción de clase infinita y sus reglas de manipulación se hallan en las raíces de gran parte de la matemática superior, haríamos bien en recapacitar si cualquier progreso posterior en al ámbito de cálculo -o incluso en cualquier otra rama de la matemática avanzada- únicamente llegará a consolidarse tras dilucidar sus conexiones con la teoría del infinito.

Por último, hay quien podría subrayar la curiosa coincidencia por la cual dos parejas formadas respectivamente por un inglés y un francés solventaron los problemas de fundamentos suscitados por la obra de su antecesor. Así como Newton precedió a Cauchy, Dirac abrió paso a los logros de Schwartz, si bien las personalidades de todos ellos apenas encuentran semejanzas. Tímido e introvertido, el temperamento de Dirac jamás alcanzó el grado de hosquedad que se atribuye a Newton, ni el conservadurismo de que siempre hizo gala Cauchy encuentra el menor parangón en la ideología radical de Schwartz. Pero a todos ellos unía, eso sí, un amor compartido por la búsqueda de la verdad que jamás desfalleció, pese a no contar con otro sustento que su propia ejecutoria. 


\section{Bibliografía}

Berkeley, G. (1951), The Works of George Berkeley, vol. 4, London: Nelson \& Sons.

Birkhoff, G. (ed.) (1973), A Sourcebook in Classical Analysis, Harvard: Harvard University Press.

Bochner, S. (1932), Vorlesungen über Fouriersche Integrale, Leipzig: Akadamie-Verlag.

Bochner, S. (1991), El papel de la matemática en el desarrollo de la ciencia, Madrid: Alianza Universidad.

Bombal, F. (2003), "Laurent Schwartz, el matemático que quería cambiar el mundo”, La Gaceta de la RSME 6 (1): 177-201.

Bolzano, B (1810), Beyträge zu einer begründeteren Darstellung der Mathematik. Erste Lieferung, Prague: Caspar Widtmann.

Bolzano, B. (1817), Die drey Probleme der Rectification, Leipzig: Paul Gotthelf Kummer.

Bottazzini, U. (1986), The Higher Calculus: A History of Real and Complex Analysis from Euler to Weierstrass, New York: Springer.

Boyer, C.B. (1959), The History of the Calculus and Its Conceptual Development, New York: Dover.

Bremermann, H. J. y L. Durand (1961), "On Analytic Continuation, Multiplication and Fourier Transformations of Schwartz Distributions”, Journal of Mathematical Physics 2 (2): 240-258.

Brezis, H. y F. Browder (1998), "Partial Differential Equations in the 20th Century", Advances in Mathematics 135: 76-144.

Bunge, M. (2006), A la caza de la realidad, Barcelona: Gedisa.

Burtt, E. A. (1925), The Metaphysical Foundations of Modern Physical Science, New York: Doubleday.

Carmichael, R. D. y D. Mitrovic (1989), Distributions and Analytical Functions, New York: J. Wiley \& Sons.

Cauchy, A. L. (1821), Cours d'Analyse de l'École Royale Polytechnique, Paris: Imprimerie Royale.

Cauchy, A. L. (1823), Résumé des Leçons données a l'École Royale Polytechnique sur l'Calcul Infinitésimal, Paris: Imprimerie Royale.

Child, J. M. (1920), The Early Mathematical Manuscripts of Leibniz, London: Open Court.

Colyvan, M. (2001), The Indispensability of Mathematics, Oxford: Oxford University Press.

Demidov, A. S. (2001), Generalized Functions in Mathematical Physics, New York: Nova Science.

Dieudonné, J. (1981), History of Functional Analysis, Amsterdam: North-Holland.

Dirac, P. A. M. (1926), "On the Theory of Quantum Mechanics”, Proceedings of the Royal Society London A 112: 661-677.

Dirac, P. A. M. (1958), The Principles of Quantum Mechanics, $4^{\text {th }}$ ed. revised, Oxford: Clarendon Press.

Dirichlet, P. G. L. (1830), "Solution d'une question relative à la théorie mathématique de la chaleur", Journal für die reine und angewandte Mathematik 5: 287-295.

Egorov, Y. (1990), “A Contribution to the Theory of Generalized Functions”, Russian Mathematical Surveys 45 (5): 1-49.

Fisher, G. (1978), “Cauchy and the Infinitely Small”, Historia Mathematica 5: 313-331.

Fourier, J. (1822), Théorie analytique de la chaleur, Paris: Gauthiers-Villars. 
Gårding, L. (1991), Some Points of Analysis and Their History, Providence: American Mathematical Society.

Gel'fand, I. M. y Shilov, G. E. (1964), Generalized Functions, vol. I, New York: Academic Press.

González-Urbaneja, P.M. (1992), Las raíces del cálculo infinitesimal en el siglo XVII, Madrid: Alianza Universidad.

Grabiner, J. V. (1978), “The Origins of Cauchy's Theory of the Derivative”, Historia Mathematica 5: 379-409.

Grabiner, J. V. (1981), The Origins of Cauchy's Rigorous Calculus, New York: Dover.

Grattan-Guinness, I. (1970a), The Development of the Foundations of Mathematical Analysis from Euler to Riemann, Massachusetts: MIT Press.

Grattan-Guinness, I. (1970b), "Bolzano, Cauchy and the 'New Analysis' of the Early Nineteenth Century", Archive for History of Exact Sciences 6 (5): 372-400.

Halperin, I. y L. Schwartz (1952), Introduction to the Theory of Distributions, Toronto: Toronto University Press.

Heaviside, O. (1893), "Operators in Mathematical Physics", Proceedings of the Royal Society of London-Series A 52: 504-529.

Heaviside, O. (1894), "Operators in Mathematical Physics", Proceedings of the Royal Society of London-Series A 53: 105-143.

Janiak, A. (2008), Newton as Philosopher, New York: Cambridge University Press.

Kirchhoff, G. R. (1878), Vorlesungen über mathematische Physik, Leipzig: Teubner.

Kirchhoff, G. R. (1882) "Zur Theorie des Lichtstrahlen”, Sitzungsberichte der Königlichen Preussische Akademie der Wissenschaften 18: 641-669.

Lagrange, J.- L. (1813), Oeuvres, vol. 9, Paris: Gauthier-Villars.

Laugwitz, D. (1989), "Definite Values of Infinite Sums: Aspects of the Foundations of Infinitesimal Analysis Around 1820", Archive for History of Exact Sciences 39: 195-245.

Levine, I. N. (1977), Quimica cuántica, Madrid: Editorial AC.

Lightstone, A. H. y K. Wong (1975), “Dirac Delta Functions via Nonstandard Analysis”, Canadian Mathematical Bulletin 18 (5): 759-762.

Lützen, J. (1979), "Heaviside's Operational Calculus and the Attempts to Rigorize It", Archive for History of Exact Sciences 21: 161-200.

Lützen, J. (1982), The Prehistory of the Theory of Distributions, New York: Springer.

Luzin, N. N. (2003), "Función” (versión castellana de J. M. Almira y D. Arcoya), La Gaceta de la RSME 6 (2): 413-436.

Mikusiński, J. (1983), Operational Calculus-Vol. I, $2^{\text {nd }}$ ed., New York: Pergamon Press.

Newton, I. (1726), Naturalis Philosophiae Principia Mathematica (Editio tertia), Londini: Regiae Societatis Typographos.

Newton, I. (1736), The Method of Fluxions and Infinite Series with Its Application to the Geometry of Curve-lines, London: H. Woodfall.

Newton, I. (1997), Principios matemáticos de filosofía natural, Madrid: Tecnos.

Newton, I. (2004), Philosophical Writings (ed. por A. Janiak), Cambridge: Cambridge University Press. 
Roy, R. (1990), "The Discovery for the Series Formula for $\pi$ by Leibniz, Gregory and Nilakantha”, Mathematics Magazine 63 (5): 291-306.

Rusnock, P. (2000), Bolzano's Philosophy and the Emergence of Modern Mathematics, Rodopi: Amsterdam.

Russ, S. B. (1980), “A Translation of Bolzano’s Paper On the Intermediate Value Theorem”, Historia Mathematica 7: 156-186.

Russ, S. (2006), The Mathematical Works of Bernard Bolzano, Oxford/New York: Oxford University Press.

Russell, B. (1969), Análisis de la materia, Madrid: Taurus.

Schwartz, L. (1950-1951), Théorie des distributions, Paris: Hermann.

Schwartz, L. (1954-1955), "Espaces de fonctions différentiables à valeurs vectorielles”, Journal of Analytical Mathematics 4: 88-148.

Schwartz, L. (1957/1959), "Théorie des distributions à valeurs vectorielles I”, Annales de l'Institut Fourier 7: 1-141; 8: 1-209.

Schwartz, L. (1959), “Théorie des distributions à valeurs vectorielles II”, Annales de l'Institut Fourier 8: 1-209.

Schwartz, L. (1968), Applications of Distributions to the Theory of Elementary Particles in Quantum Mechanics, New York: Gordon \& Breach Science Publ.

Sellés, M. (2006), "La paradoja de Galileo”, Asclepio 58 (1): 113-148.

Struik, D. (ed.) (1969), A Source Book in Mathematics, 1200-1800, Harvard: Harvard University Press.

Thayer, H. S. (ed.) (1953), Newton's Philosophy of Nature, New York: Hafner-Macmillan.

Thomae, J. K. (1875), Einleitung in die Theorie der bestimmten Integrale, Halle: Louis Nebert.

Tréves, F. (1966), Linear Partial Differential Equations with Constant Coefficients, New York: Gordon \& Breach Science Publ.

Von Neumann, J. (1932), Mathematical Foundations of Quantum Mechanics (English translation, 1955), Princeton: Princeton University Press.

Weierstrass, K. (1895), Mathematische Werke, vol. 2, Berlin: Knoblauch.

Weierstrass, K. (1988), Ausgewählte Kapitel aus der Funktionenlehre (ed. por R. Siegmund-Schultze), Leipzig: Teubner.

Zermelo, E. (1904), "Beweis, daß jede Menge wohlgeordnet werden kann”, Mathematische Annalen 59: 514-516. 
